\title{
Resultados maternos e perinatais em gestações com placenta prévia com e sem acretismo em maternidade terciária
}

\section{Maternal and perinatal outcomes in pregnancies with placenta previa with and without accreta at a tertiary center}

Erika Roberta de Lima ${ }^{1}$. Helvécio Neves Feitosa ${ }^{1,2}$. Francisco Edson de Lucena Feitosa ${ }^{1}$. Francisco Herlânio Costa Carvalho ${ }^{1}$.

1 Maternidade-Escola Assis Chateaubriand, Universidade Federal do Ceará (UFC). 2 Universidade de Fortaleza (UNIFOR).

RESUMO

Objetivo: Avaliar os resultados maternos e perinatais nos casos de placenta prévia com e sem acretismo na Maternidade-Escola Assis Chateaubriand-UFC. Metodologia: Estudo transversal, quantitativo, restrospectivo, de 19 pacientes com placenta prévia, em 2014. Na análise bivariada, utilizou-se o teste exato de Fisher e o test t-student para as variáveis categóricas e contínuas, respectivamente. Considerou-se nível de significância quando $\mathrm{p}<0,05$. Resultados: As prevalências de placenta prévia e acretismo foram $0,46 \%$ e $0,1 \%$, respectivamente. No grupo de pacientes com acretismo, as médias de idade materna e paridade foram maiores (29,5 x 26 anos; $2 \times 1$, respectivamente); apresentou pelo menos uma cesárea prévia ( $\mathrm{p}=0,031)$; houve maior necessidade de hemotransfusões $(50 \%$ x 6,7\%), de internações em UTI materna (25\% x 0), lesão vesical (25\% x 0) e maior necessidade de histerectomia $(\mathrm{p}=0,023)$. Conclusões: Placenta prévia é associada a aumento da morbidade materna e perinatal. Antecedente de cesariana aumentou a chance de acretismo, bem como a necessidade de histerectomia.

Palavras-chave: Placenta prévia. Placenta acreta. Hemorragia. Avaliação de resultados da assistência ao paciente.

\section{ABSTRACT}

Objective: To evaluate maternal and perinatal outcomes in cases of placenta previa with and without accreta at Maternity School Assis Chateaubriand-UFC. Methods: Cross-sectional study, quantitative, restrospective of 19 patients with placenta previa, in 2014. In the bivariate analysis, we used Fisher's exact and the t-student tests for categorical and continuous variables, respectively. It was considered a significance level of $\mathrm{p}<0.05$. Results: The prevalence of placenta previa and accreta were $0.46 \%$ and $0.1 \%$, respectively. In the group of patients with accreta, the mean maternal age and parity were higher (29.5 x 26 years, $2 x 1$ respectively); had at least one previous cesarean section $(p=0.031)$; there was a greater need for blood transfusions $(50 \%$ x $6.7 \%)$, of hospitalizations in maternal ICU (25\% vs. 0$)$, bladder injury $(25 \%$ vs. 0$)$ and greater need for hysterectomy ( $\mathrm{p}$ $=0.023)$. Conclusions: Placenta previa is associated with increased maternal and perinatal morbidity. Previous cesarean section increased the chance of accreta, as well as the need for hysterectomy.

Keywords: Placenta previa. Placenta accreta. Hemorrhage. Patient outcome assessment.

Autor correspondente: Erika Roberta de Lima, Universidade Federal do Ceará. E-mail: e.erika.lima@gmail.com. Conflito de interesses: Não há qualquer conflito de interesses por parte de qualquer um dos autores.

Recebido em: 02 Mai 2015; Revisado em: 20 Mai 2015; Aceito em: 23 Mai 2015. 


\section{INTRODUÇÃO}

A Placenta prévia constitui entidade patológica de modalidade anômala de inserção da placenta e pode cursar concomitantemente com o acretismo placentário nos casos de desenvolvimento precário da decídua, em geral, no segmento uterino inferior. ${ }^{1}$ Compõem causas significativas de morbidade e mortalidade materna e neonatal. ${ }^{2}$

A placenta prévia acomete uma em cada 250 gestações. ${ }^{3}$ Ressalta-se ainda, nos últimos anos, o aumento da sua incidência entre as pacientes com cicatriz uterina decorrente de cesariana. ${ }^{4,5}$ Cesárea única praticamente dobra o risco de desenvolvimento de placenta prévia na gestação seguinte de $0,38 \%$ para $0,63 \%$, aumentando ainda mais com aumento do número de cesarianas prévias. ${ }^{5,6}$

Do ponto de vista do impacto fetal e neonatal, as gestações complicadas por placenta prévia têm sido associadas ao maior número de nascimentos pretermos. ${ }^{3,7}$ Verificaram-se ainda outras associações tais como níveis mais altos de anomalias fetais, atraso no neurodesenvolvimento e risco de síndrome de morte súbita infantil. ${ }^{8}$ Além disso, constatou-se alto risco para Restrição de Crescimento Intrauterino (RCIU), fator de risco significativo para mortalidade perinatal. $^{7,9} \mathrm{O}$ sangramento transvaginal secundário à placenta prévia é responsável por níveis de mortalidade perinatal de até $2,3 \%{ }^{2}$

O acretismo placentário ocorre em um a cada 1000 partos. É considerada complicação gestacional severa por conta do risco significativo de cursar com hemorragias maciças e potencialmente fatais. ${ }^{10}$ Há estudos apontando que o distúrbio pode constituir até $51,1 \%$ das indicações cirúrgicas de histerectomia de emergência. ${ }^{11}$ Nos Estados Unidos, os níveis de histerectomia periparto por acretismo placentário aumentou em 20\% de 1994 a 2007. ${ }^{12}$ A perda sanguínea durante o parto pode atingir de 3 a 5 litros nos casos mais severos de acretismo. ${ }^{13}$ Alguns estudos apontam que a morbidade materna poderia atingir até $60 \%$ das mulheres acometidas por acretismo .placentário, enquanto a mortalidade poderia alcançar até $7 \%$ dos casos. ${ }^{7}$

Tendo em vista o impacto que a inserção anômala da placenta possa exercer sobre os indicadores em obstetrícia, este estudo objetiva avaliar diferenças nas apresentações clínicas e os resultados maternos e perinatais nos casos de placenta prévia com e sem acretismo placentário na Maternidade-Escola Assis Chateaubriand-UFC, em 2014.

\section{METODOLOGIA}

Trata-se de estudo transversal, quantitativo, cujos dados foram obtidos de forma retrospectiva por meio de revisão dos prontuários de pacientes diagnosticadas com placenta prévia, após idade gestacional de 28 semanas, que permaneceram internadas no Serviço de Medicina Materno Fetal da Maternidade-Escola Assis Chateaubriand no ano de 2014. Constatou-se o internamento de 22 pacientes, entretanto três prontuários não foram localizados e, assim, a amostra contabilizou 19 pacientes, as quais foram divididas em dois grupos: casos de placenta prévia sem acretismo, com 15 pacientes, e casos de placenta prévia com acretismo, com quatro pacientes. O diagnóstico de acretismo foi realizado retrospectivamente pelo achado anatomopatológico ou, na ausência deste, pela descrição cirúrgica. Não se considerou a suspeita ultrassonográfica ou da ressonância magnética para classificação entre os dois grupos.

Foram preenchidas fichas referentes a cada paciente, em que as variáveis a serem estudadas viabilizaram a comparação entre os grupos quanto aos dados clínico-obstétricos e os desfechos maternos e perinatais.

As variáveis clínico-obstétricas de interesses foram: idade materna (em anos), número de gestações, partos e abortamentos, antecedente de cesariana e curetagem uterina, classificação da placenta prévia (total, parcial ou marginal), forma de diagnóstico (clínico e/ou ecográfico) e a idade gestacional na apresentação clínica (em semanas). As variáveis desfechos foram: idade gestacional no parto (em semanas), via de parto (vaginal ou abdominal), presença de lesão vesical, necessidade de histerectomia, necessidade de hemotransfusão, internamento em UTI materna e neonatal, tempo de permanência nessas UTIs, peso ao nascer (em gramas) e escore de Apgar no primeiro minuto de vida. Considerou-se para idade gestacional no parto, o valor do Capurro somático, em virtude da dificuldade de registro em prontuário de ultrassom precoce.

Foram seguidos todos os princípios éticos para pesquisa com seres humanos respeitando a resolução 466/2012 do Conselho Nacional de Saúde. O projeto de pesquisa foi aprovado, sem restrições, com número de parecer 924.779 pelo Comitê de Ética em Pesquisa da Maternidade-Escola Assis Chateaubriand - Universidade Federal do Ceará.

A amostragem foi obtida por conveniência (todas as pacientes internadas na MEAC-UFC no ano de 2014 com informações disponíveis em prontuários). Os dados foram expressos em média e desvio padrão para as variáveis contínuas e em proporções para as variáveis categóricas. $\mathrm{Na}$ análise bivariada, utilizou-se o teste exato de Fisher e o teste t-student para as variáveis categóricas e contínuas, respectivamente. Considerou-se nível de significância quando $\mathrm{p}<0,05$. A análise estatística foi realizada com auxílio do programa STATA versão 12 (College Station, TX, USA).

\section{RESULTADOS}

O estudo incluiu 19 pacientes com diagnóstico clínico e/ou ultrassonogáfico de placenta prévia internadas na Maternidade-Escola Assis Chateaubriand no ano de 2014. No referido ano, o número total de partos realizados na MEAC foi de 3.911; portanto, a prevalência de placenta prévia foi de $0,46 \%$ do total de nascimentos. Nesse mesmo período, os casos complicados por acretismo placentário constituíram $0,1 \%$ dos nascimentos e $21,1 \%$ dos casos de placenta prévia.

Entre as pacientes portadoras de placenta prévia não associada ao acretismo $(n=15)$, observou-se que a idade 
média foi de 29,5 anos $( \pm 7,6)$, o número médio de gestações foi de quatro $( \pm 2,7)$ e da quantidade de partos de dois $( \pm$ 1,8). Nesse grupo, $53,3 \%$ das pacientes não apresentavam história de cesárea anterior nem curetagem, enquanto 33,3\% já haviam sido submetidas apenas a curetagem; 6,6\% delas apresentavam cesárea anterior única e outros $6,6 \%$ apresentaram duas ou mais cesáreas anteriores (Tabela 1).

Tabela 1 - Comparação das características clínico-obstétricas entre as pacientes com placenta prévia com e sem acretismo. MaternidadeEscola Assis Chateaubriand-UFC, 2014.

VARIÁVEIS SEM ACRETISMO COM ACRETISMO p

Idade Materna, anos (média \pm DP)

Número de Gestações (média \pm DP)

Número de Partos (média \pm DP)

Número de Abortos (média \pm DP)

Cesárea Anterior (n, \%)

Curetagem Prévia (n, \%)

Classificação da PP

Centro-Total

Centro-Parcial

Marginal

Diagnóstico Clínico (STV)

IG no primeiro STV

Diagnóstico Ecográfico (assintomáticas)

$\begin{array}{lcc}29,5( \pm 7,6) & 26( \pm 3,6) & 0,608^{*} \\ 4( \pm 2,7) & 3( \pm 0,4) & 0,089^{*} \\ 2( \pm 1,8) & 1( \pm 0,5) & 0,201^{*} \\ 0,66( \pm 1,5) & 0,75( \pm 0,5) & 0,611^{*} \\ 1(6,7 \%) & 4(100 \%) & \mathbf{0 , 0 3 1 * *} \\ 5(33,3 \%) & 3(75 \%) & 0,392^{* *}\end{array}$

$9(60 \%)$

$4(100 \%)$

$0,628 * *$

$2(13 \%)$

$3(20 \%)$

$14(93,3 \%)$

$4(100 \%)$

$0,942 * *$

27

$0,102 *$

$1(6,7)$

$0,821 * *$ 
Tabela 2 - Comparação dos resultados maternos e perinatais entre as pacientes com placenta prévia com e sem acretismo. Maternidade-Escola Assis Chateaubriand-UFC, 2014.

VARIÁVEIS

$\begin{array}{ll}\text { SEM } & \text { COM } \\ \text { ACRETISMO } & \text { ACRETISMO }\end{array}$

IG no Parto, semanas (média \pm DP)

$32,5( \pm 9,9)$

$35,7( \pm 1,5)$

$0,595^{*}$

Via de Parto

$0,668 * *$

Abdominal (n, \%)

Vaginal (n, \%)

Histerectomia (n, \%)

Lesão Vesical (n, \%)

Hemotransfusão (n, \%)

Internamento em UTI materna (n, \%)

Tempo de internamento UTI, dias (média \pm DP)

Peso ao nascer, gramas (média \pm DP)

Apgar 1 minuto (média \pm DP)

Apgar $\leq 7$ (n, \%)

Internamento em UTI Neonatal (n, \%)

Tempo de internamento UTI Neo, dias (média \pm DP)

$$
\begin{aligned}
& 12(80 \%) \\
& 2(20 \%)
\end{aligned}
$$

0

0

$1(6,7 \%)$

0

$2658( \pm 412,6)$

$5,5( \pm 2,8)$

$8(57 \%)$

$3(23 \%)$

$21( \pm 17,7)$
$4(100 \%)$

$4(100 \%)$

$0,023 * *$

$1(25 \%)$

$0,084 * *$

$2(50 \%)$

$0,095 * *$

$1(25 \%)$

$0,081 * *$

5

$2500( \pm 344,8)$

$0,319 *$

$8,5( \pm 0,6)$

$0,469^{*}$

0

$0,182 * *$

$2(50 \%)$

$0,412 * *$

$0,487^{*}$

*Teste t-student; **Teste exato de Fisher. IG= Idade gestacional; PP=Placenta prévia; STV= Sangramento transvaginal; $\mathrm{DP}=\mathrm{Desvio}$ padrão; UTI=Unidade de Terapia Intensiva.

Quanto aos fatores neonatais, a idade média com base no Capurro foi de $32,5( \pm 9,9)$ semanas; o peso médio ao nascer de $2.658( \pm 412,6) \mathrm{g}$ e a média de Apgar $1^{\circ}$ minuto $=5,5( \pm$ 2,8). Em $23 \%$ dos RNs ( $=3)$ houve internamento em UTI neonatal cujo tempo médio foi de $21( \pm 17,7)$ dias, atribuídos a complicações decorrentes da prematuridade (Tabela 2).

No grupo com placenta prévia associada ao acretismo placentário, a idade média das pacientes foi de $26( \pm 3,59)$ anos, observando-se ainda médias de três gestações $( \pm 0)$, um parto $( \pm 0,5)$ e um aborto $( \pm 0,5)$. A sintomatologia inicial em todo o grupo foi o sangramento transvaginal iniciado em média no curso de 27 semanas de gestação (Tabela 1).

A via de parto foi cirúrgica em todos os casos, sendo que em $75 \%(n=3)$ deles o procedimento foi eletivo cuja placenta foi mantida in situ, seguida de histerectomia abdominal total. Houve um caso de cesariana emergencial em decorrência do sangramento transvaginal moderado em paciente portadora de placenta prévia centro-total cujo acretismo, embora não suspeitado ao exame ecográfico, foi detectado durante o ato cirúrgico mediante dificuldade técnica para remoção manual da placenta. Neste caso, a paciente evoluiu com choque hipovolêmico refratário durante o pós-operatório imediato, motivando a realização de histerectomia abdominal subtotal durante a primeira intervenção, e hemostasia em coto uterino durante a segunda intervenção.

Entre as complicações maternas, verificou-se lesão de bexiga em um caso (25\%), fato atribuído à dificuldade técnica durante a execução do procedimento em que se observou parede uterina anterior de aspecto infiltrativo, sugestivo de placenta percreta, estendendo-se até a porção superior da parede vesical. Assim sendo, durante extração da peça cirúrgica (útero) ocorreu lesão vesical, seguida de rafia imediata. Além disso, em metade do grupo foi necessária hemotransfusão. Uma paciente necessitou de internamento em UTI durante o pós-operatório durante cinco dias em decorrência da instabilidade hemodinâmica secundária ao choque hipovolêmico mencionado (Tabela 2). 
Quanto aos fatores neonatais constatados no grupo de pacientes com placenta prévia associada ao acretismo placentário, idade média ao Capurro=35,7 $( \pm 1,5)$ semanas; peso médio ao nascer $=2.500( \pm 344,8) \mathrm{g}$; Apgar médio $1^{\circ}$ minuto $=8,5( \pm 0,6)$ e tempo de internamento médio de $16( \pm$ 11,3) dias. Não houve morte perinatal (Tabela 2).

$\mathrm{Na}$ análise bivariada para comparação entre os grupos, houve diferença estatisticamente significante somente para presença de cesárea prévia $(\mathrm{p}=0,031)$, em que $100 \%$ das pacientes do grupo com acretismo placentário apresentava esse dado (versus 6,7\% no grupo sem acretismo) (Tabela 1). Observouse idade gestacional de apresentação clínica maior (32 semanas) no grupo sem acretismo e menor (27 semanas) no grupo com acretismo, mas sem diferença estatistica $(p=0,102)$. Para os desfechos maternos, houve diferença significativa para a necessidade de histerectomia (100\% no grupo com acretismo e nenhum caso no grupo sem acretismo, $p=0,023)$. Não houve significância estatística para nenhum dos desfechos neonatais. Verificaram-se escores médios de Apgar de primeiro minuto maiores no grupo com acretismo (8,5 versus 5,5); no entanto, sem significância estatística $(p=0,469)$. Quando se considerou proporção de Apgar $<7$ no primeiro minuto também não houve diferença $(\mathrm{p}=0,182)$ (Tabela 2).

Não houve caso de morte materna, restrição de crescimento, malformação congênita ou morte neonatal precoce.

\section{DISCUSSÃO}

A prevalência de placenta prévia na Maternidade-Escola Assis Chateaubriand durante 2014 foi $0,46 \%$, o que está de acordo com Tuzovic et al., ${ }^{14}$ que afirmaram que a incidência nesses casos poderia variar entre $0,3-0,8 \%$ das gestações em todo o mundo.

Quanto ao acretismo placentário, a prevalência pode abranger faixa entre $0,04-0,9 \%$ de acordo com Eshkoli et al., ${ }^{10}$ o que apresentou concordância com a realidade de nosso serviço, onde o valor encontrado foi de $0,1 \%$.

Idade materna avançada e multiparidade têm sido associadas com altos riscos de placenta prévia. ${ }^{15}$ No grupo de pacientes com placenta prévia sem acretismo, a idade materna média, o número médio de gestações bem como o de partos foram maiores em relação ao outro grupo; no entanto, sem diferença estatística.

Ainda, que não se tenha observado diferença significativa quanto ao número de gestações e de partos, a multiparidade é considerada como fator de risco importante tanto para placenta prévia como para acretismo placentário. ${ }^{9}$

Em mulheres com placenta de inserção baixa associada à cirurgia uterina anterior, o ultrassonografista deve ser mais cauteloso ao investigar acretismo durante o exame. ${ }^{16} \mathrm{O}$ risco de acretismo na vigência de placenta prévia aumenta conforme o número de cesáreas, sendo de $11 \%$ após uma, $40 \%$ após duas, $61 \%$ após três e $67 \%$ após quatro ou mais. ${ }^{17}$ Assim sendo, no grupo de placenta prévia sem acretismo, apenas $6,6 \%$ apresentaram uma cesárea anterior, enquanto no grupo com acretismo concomitante $100 \%$ das pacientes contabilizavam ao menos uma cesárea em gestações anteriores. Desta forma, a variável cesárea anterior foi mais comum entre as pacientes portadoras de acretismo concomitante com a placenta prévia.

As internações são justificadas, em geral, por conta do sangramento transvaginal, ${ }^{16}$ o que está de acordo com nosso estudo, em que apenas 6,7\% das pacientes portadoras de placenta prévia sem acretismo permaneceram assintomáticas, sendo o diagnóstico obtido por meio de exame ecográfico durante o rastreio pré-natal, enquanto na grande maioria deste grupo (93,3\%), o diagnóstico ocorreu por meio de sangramento transvaginal assim como no grupo com acretismo associado (100\%).

Observou-se ainda que o sangramento foi mais precoce entre as pacientes com acretismo concomitante, iniciando em torno de 27 semanas em média, enquanto entre aquelas portadoras unicamente de placenta prévia a sintomatologia iniciou-se mais tardiamente, em torno de 32 semanas; não houve, no entanto, diferença com significância estatística. Segundo o estudo retrospectivo de coorte realizado por Lam et al., ${ }^{18}$ dentre 252 gestantes portadoras de placenta prévia, 159 delas evoluíram com sangramento transvaginal antes do parto que transcorrera, em geral, com menos de 34 semanas. Assim sendo, o sangramento transvaginal compõe a principal manifestação de ambas as patologias, de modo que tende a iniciar-se mais cedo entre os casos de acretismo concomitante.

Uma vez conhecida relação entre a placenta acreta e a combinação de placenta prévia e cesárea anterior, ${ }^{19}$ sendo detectada imagem sugestiva de acretismo placentário ao ultrassom ou à ressonância magnética, deve ser planejada cirurgia eletiva do tipo cesárea-histerectomia. Embora os níveis de mortalidade sejam menores em cirurgias eletivas que naquelas de emergência, a cesárea-histerectomia por placenta acreta, independente de situação emergencial ou não, tem sido a escolha. ${ }^{20}$

No grupo de pacientes portadoras de placenta prévia associada ao acretismo placentário, em $75 \%$ delas já houve programação para histerectomia, sem tentativa de retirada da placenta, pela suspeita aos exames de imagem do acretismo. De acordo com Eshkoli et al., ${ }^{10}$ a histerectomia é uma cirurgia radical, propiciando redução da morbidade materna e tratamento definitivo para o acretismo.

Essa conduta, atualmente adotada em nosso serviço, pode justificar os melhores valores de Apgar encontrados no grupo das pacientes com acretismo. Os recém-nascidos apresentaram melhores condições de vitalidade ao primeiro minuto de vida. O pequeno número de casos no grupo do acretismo pode ser responsável pela falta de significância estatística para essa variável. Com o aumento dos participantes no grupo do acretismo, talvez pudéssemos comprovar essa diferença.

A presença de acretismo placentário pode ser responsável, ainda, por outros impactos na morbidade materna, tais como necessidade de hemotransfusões (50\% x 6,7\%); internamento 
em UTI materna $(25 \%$ x 0$)$ bem como lesão vesical intraoperatória $(25 \% \quad x \quad 0)$. Novamente, a falta de significância estatística pode ser atribuída ao pequeno número de participantes nesse grupo. De acordo com Eshkoli et al., ${ }^{10}$ o acretismo placentário pode ser significativamente associado com resultados gestacionais adversos tais como sangramento transvaginal de segundo trimestre, hemorragia pós-parto, transfusão sanguínea materna, bem como admissão em UTI entre outros.

A necessidade de internamento em UTI materna de uma paciente com placenta prévia centro-total (PPCT) pode ser atribuída ao diagnóstico falso negativo para acretismo ao ultrassom transvaginal durante a internação, aliado ao sangramento transvaginal moderado em condições emergenciais que impuseram a necessidade de resolução da gestação. Durante a cirurgia em questão, tentou-se remover a placenta, a qual saiu após relativo grau de dificuldade, sendo aventada a suspeita de acretismo no intraoperatório. Essa suspeita confirmou-se no pós-operatório imediato, quando a paciente evoluiu com choque hipovolêmico por duas vezes, requerendo a necessidade de realizar duas reabordagens cirúrgicas, sendo que na primeira foi realizada histerectomia subtotal, enquanto na segunda houve hemostasia de coto do colo uterino.

No grupo de pacientes com placenta prévia associada ao acretismo, em que a cesariana foi seguida por histerectomia, verificou-se ainda lesão vesical em $25 \%$ dos casos como complicação intraoperatória, atribuída à possível extensão do componente percreta placentário até a porção superior da bexiga. Vale destacar que no outro grupo não se constataram lesões em órgãos pélvicos adjacentes ao útero.

Com relação aos parâmetros neonatais, verificou-se que, no grupo de pacientes com placenta prévia e acretismo placentário, os recém-nascidos apresentaram maior idade média ao nascer $(35,7$ x 32,5 semanas); melhor média de Apgar de primeiro minuto $(8,5 \times 5,5)$, valendo ressaltar que nenhum recém-nascido deste grupo obteve Apgar < 7. Destaca-se que a prematuridade ao nascer foi fator prevalente tanto para placenta prévia como para acretismo placentário.

Ressalte-se que em 50\% dos casos $(n=2)$ de acretismo concomitante houve necessidade de internação do recémnascido em UTI neonatal, ainda que com menor tempo médio de internamento neste setor $16( \pm 11,3)$ dias. Por outro lado, em apenas $23 \%(n=3)$ dos casos de placenta prévia sem

\section{REFERÊNCIAS}

1. Cunningham FG, Leveno KJ, Bloom SL, Hauth JC, Rouse DJ, Spong CY. Obstetrícia de Williams. 23. ed. Porto Alegre: AMGH editora; 2012. Capítulo 35, Hemorragia obstétrica; p. 757-803.

2. Crane JM, Van den Hof MC, Doods L, Armson BA, Liston R. Neonatal outcomes with placenta previa. Obstet Gynecol. 1999;93(4):541-4.

3. Fishman SG, Chasen ST, Maheshwari B. Risk factors for preterm delivery with placenta previa. J Perinat Med. 2011;40(1):39-42. acretismo os recém-nascidos precisaram permanecer internados em UTI neonatal durante tempo médio maior de $21( \pm 17,7)$ dias.

As limitações do estudo são várias, entre elas pode-se citar: estudo retrospectivo (quando pode haver perda de informações pela ausência de registro adequado em prontuário) e pequeno número de participantes para apresentar poder de generalizar suas conclusões. No entanto, a população estudada se assemelha a várias outras consideradas em ambiente de assistência materno infantil, especialmente no Brasil onde os índices de cesariana já se encontram acima de $50 \%$ dos nascimentos e as taxas de natalidade entre dois e três filhos.

A proposta do estudo foi avaliar resultados dessa patologia ao longo de um ano. Provavelmente uma séria histórica de pelo menos seis anos de seguimento venha dar melhores respostas à questão de quão diferentes são os quadros de placenta prévia com ou sem acretismo, especialmente para aquelas variáveis que se mostraram diferentes, mas sem significância estatística, por exemplo: idade gestacional do primeiro sangramento transvaginal e escores de Apgar melhores no grupo do acretismo. A vitalidade neonatal encontrada melhor no último grupo pode ter sido explicada pela adoção da programação de cesariana-histerectomia, sem tentativa de delivramento, frente às suspeitas imaginológicas de acretismo adotada pelo serviço no último ano. Espera-se, agora, comparar esses mesmos resultados maternos e perinatais antes e após essa mudança de conduta nos casos suspeitos de acretismo - de preferência com estudos de coorte prospectivos. Espera-se também aumentar a casuística para expandir as conclusões.

Apesar do pequeno número de participantes nesse estudo retrospectivo, pode-se constatar que, mesmo em centro de referência para atenção à saúde materna e neonatal, o achado de placenta prévia (com ou sem acretismo) é associado a aumento da morbidade materna (necessidade de internamento em UTI, hemotransfusões, lesões de orgãos, operações cesarianas e histerectomias) e perinatal (prematuridade, menores escores de Apgar, internamento em UTI neonatal). Quando se comparou os grupos, verificou-se que na presença de placenta prévia o achado de antecedente de cesariana aumentou a chance da coexistência de acretismo placentário, bem como a necessidade de histerectomia intra ou pós-operatória.

4. Hemminki E, Merilainen J. Long-term effects of cesarean sections: ectopic pregnancies and placental problems. Am J Obstet Gynecol. 1996;174(5):1569-1574.

5. Orbach A, Levy A, Wiznitzer A,Mazor M, Holcberg G, Sheiner E. Peripartum cesarean hysterectomy: critical analysis of risk factors and trends over the years. J Matern Fetal Neonatal Med. 2011;24(3):480-4.

6. Monica G, Lilja C. Placenta previa, maternal smoking and recurrence risk. Acta Obstet Gynecol Scand. 1995;74(5):341-5. 
7. Eller AG, Porter TF, Soisson P, Silver RM. Optimal management strategies for placenta accreta. BJOG. 2009;116(5): 648-54

8. Li DK, Wi S. Maternal placental abnormality and the risk of sudden infant death syndrome. Am J Epidemiol. 1999;149(7):608611.

9. Harper LM, Odibo AO, Macones GA, Crane JP, Cahill AG. Effect of placenta previa on fetal growth. Am J Obstet Gynecol. 2010;203(4):330.e1-5.

10. Eshkoli T, Weintraub AY, Sergienko RB, Sheiner E. Placenta accreta: risk factors, perinatal outcomes, and consequences for subsequent births. Am J Obstet Gynecol. 2013;208(3):219.e1-7.

11. Daskalakis G, Anastasakis E, Papantoniou N, Mesogitis S, Theodora M, Antsaklis A. Emergency obstetric hysterectomy. Acta Obstet Gynecol Scand. 2007;86(2):223-7.

12. Bateman BT, Mhyre JM, Callaghan WM, Kuklina EV. Peripartum hysterectomy in the United States: nationwide 14 year experience. Am J Obstet Gynecol. 2012; 206(1):63.e1-8.

13. Hudon L, Belfort MA, Broome DR. Diagnosis and management of placenta percreta: a review. Obstet Gynecol Surv. 1998;53(8): 509-17.

14. Tuzovic L. Complete versus incomplete placenta previa and obstetric outcome. Int J Gynaecol Obstet. 2006;93(2):110-7.
15. Rao KP, Belogolovkin V, Yankowitz J, Spinnato JA. Abnormal placentation: evidence-based diagnosis and management of placenta previa, placenta accreta, and vasa previa. Obstet Gynecol surv. 2012;67(8):503-19.

16. Chantraine F, Langhoff-Roos J. Abnormally invasive placentaAIP. Awareness and pro-active management is necessary. Acta Obstet Gynecol Scand. 2013;92(4):369-71.

17. Clark SL, Koonings PP, Phelan JP. Placenta previa/accreta and prior cesarean section. Obstet Gynecol. 1985;66(1):89-92.

18. Lam CM, Wong SF, Chow KM, Ho LC. Women with placenta praevia and antepartum haemorrhage have a worse outcome than those who do not bleed before delivery. J Obstet Gynaecol. 2000;20(1):27-31.

19. Shamshirsaz AA, Fox KA, Salmanian B, Diaz-Arrastia CR, Lee W, Baker BW et al. Maternal morbidity in patients with morbidly adherent placenta treated with and without a standardized multidisciplinary approach. Am J Obstet Gynecol. 2015;212(2):218.e1-9.

20. Matsubara S, Kuwata T, Usui R, Watanabe T, Izumi A, Ohkuchi $\mathrm{A}$, et al. Important surgical measures and techniques at cesarean hysterectomy for placenta previa accreta. Acta Obstet Gynecol Scand. 2013;92(4):372-7.

\section{Como citar:}

Lima ER, Feitosa HN, Feitosa FE, Carvalho FH. Resultados maternos e perinatais em gestações com placenta prévia com e sem acretismo em maternidade terciária. Rev Med UFC. 2015 jan-jun;55(1):18-24. 